\title{
On the modeling of planetary plasma environments by a fully kinetic electromagnetic global model HYB-em
}

\author{
V. Pohjola ${ }^{1,2}$ and E. Kallio ${ }^{1}$ \\ ${ }^{1}$ Finnish Meteorological Institute, Helsinki, Finland \\ ${ }^{2}$ Department of Mathematics, University of Helsinki, Finland \\ Received: 17 October 2009 - Revised: 14 February 2010 - Accepted: 5 March 2010 - Published: 15 March 2010
}

\begin{abstract}
We have developed a fully kinetic electromagnetic model to study instabilities and waves in planetary plasma environments. In the particle-in-a-cell (PIC) model both ions and electrons are modeled as particles. An important feature of the developed global kinetic model, called HYB-em, compared to other electromagnetic codes is that it is built up on an earlier quasi-neutral hybrid simulation platform called HYB and that it can be used in conjunction with earlier hybrid models. The HYB models have been used during the past ten years to study globally the flowing plasma interaction with various Solar System objects: Mercury, Venus, the Moon, Mars, Saturnian moon Titan and asteroids. The new standalone fully kinetic model enables us to (1) study the stability of various planetary plasma regions in three-dimensional space, (2) analyze the propagation of waves in a plasma environment derived from the other global HYB models. All particle processes in a multi-ion plasma which are implemented on the HYB platform (e.g. ion-neutral-collisions, chemical processes, particle loss and production processes) are also automatically included in HYB-em model.

In this brief report we study the developed approach by analyzing the propagation of high frequency electromagnetic waves in non-magnetized plasma in two cases: We study (1) expansion of a spherical wave generated from a point source and (2) propagation of a plane wave in plasma. The analysis shows that the HYB-em model is capable of describing these space plasma situations successfully. The analysis also suggests the potential of the developed model to study both high density-high magnetic field plasma environments, such as Mercury, and low density-low magnetic field plasma environments, such as Venus and Mars.
\end{abstract}

Keywords. Magnetospheric physics (Planetary magnetospheres)

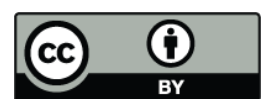

Correspondence to: E. Kallio (esa.kallio@fmi.fi)

\section{Introduction}

Space plasmas contain physical processes in a wide range of spatial and temporal scales. Local instabilities which result from global changes of the planetary plasma environments are an example of how small space scale and fast time scale processes can be linked to large scale-slow processes. Reconnection in planetary magnetospheres is an important example of instability in the Solar System. Another example is provided by electromagnetic waves, which occur at diverse spatial and temporal scales in planetary plasma environments.

Space born instruments have opened new opportunities to study instabilities and wave phenomena in the Solar System near the Earth-like planets Mercury, Venus and Mars. Electric field measurements made by Phobos-2 at Mars in early 1989 demonstrated that the plasma around the red planet contains wide range of wavelengths (e.g., Trotignon et al., 1991, 1996). Later low frequency waves at Mars were studied by using magnetometer data from Mars Global Surveyor (MGS) mission (Espley et al., 2004). The research of temporal phenomena at Mars was continued by using electron observations (Winningham et al., 2006) and energetic neutral atom measurements (Grigoriev et al., 2007) on the ongoing Mars Express (MEX) mission.

The measurements of the radio wave instrument on MEX, MARSIS, has also provided unique data from the Martian ionosphere (e.g., Nielsen et al., 2007) and the local plasma density (e.g., Duru et al., 2008) in addition to its measurements on the water content of the Martian crust. Electromagnetic waves (em-waves) carry energy and play therefore a role in the oxygen escape processes at Mars (Ergun et al., 2006) and in the Martian tail (e.g., Kallio et al., 1995). Another issue of special scientific interest is the lightning in planetary atmospheres which have been studied at Venus (Russell et al., 2007). A varying electric field has in contrast not yet been measured at Mercury and it will not be measured

Published by Copernicus Publications on behalf of the European Geosciences Union. 
by the MESSENGER mission which, after the Mercury flybys, starts making measurements at Mercury in 2011. The BepiColombo mission will however start a new phase when its MEFISTO instrument (Blomberg et al., 2006) begins to measure the Hermean wave environment around 2019.

The analysis of spatial scales from less than a meter to thousands of kilometers and time scales from less than a second to hours can not be performed by a single model. In planetary plasma physics, global scales are commonly modeled by magnetohydrodynamic (MHD) models and to lesser extent by hybrid models which have recently received an increasing amount of interest (see e.g. Brain et al., 2010). In a hybrid model ions are modeled as particles and electrons as a fluid. In a fully kinetic model also the electrons are modeled as particles and this approach has also been used in global magnetospheric research (see e.g., Nishikawa, 2001, and references therein.)

In this manuscript we describe a fully kinetic electromagnetic model, called HYB-em, which purpose is to model instabilities and electromagnetic waves in planetary plasma environments. The model is from a technical point of view largely based on an earlier three-dimensional (3-D) selfconsistent plasma modeling platform, called HYB, which has been used to study how the solar wind plasma interacts with various Solar System objects: Mercury (HYBMercury), Venus (HYB-Venus), The Moon, (HYB-Moon), Mars (HYB-Mars), Saturnian moon Titan (HYB-Titan) and asteroids (HYB-Ceres). This enables us to study instabilities and waves in 3-D space where the initial plasma and fields are derived by a global high resolution hybrid model. Incorporating the electromagnetic wave algorithm in the HYB platform enables us to study such processes in temporal and spatial scales which can not be tackled by the previous HYB models.

The difference between the HYB-em model and the other HYB models is from a physical point fundamental. HYBem model is a fully kinetic model while the other HYB models use a hybrid approach where electrons are modeled as a fluid. Consequently, HYB-em includes physics which is not included in the previous HYB models. The new model is therefore anticipated to include a rich variety of physical processes which take place at various Solar System objects, some of them not having atmospheres and where a direct plasma-surface interaction takes place (Mercury, the Moon, asteroids) and some of them having atmospheres and ionospheres (e.g. Venus, Mars, Titan).

To put the developed code into a historical perspective one may note that fully kinetic electromagnetic models based on e.g. the TRISTAN code (Buneman et al., 1980) have been used, for example, in terrestrial magnetospheric physics (e.g., Nishikawa, 2001), plasma instability studies (e.g., Sakai et al., 1998) and studies of collisionless shocks (e.g., Spitkovsky, 2008) while the KEMPO (Kyoto University Electromagnetic Particle Code) code has been studied, for example, electromagnetic and electrostatic waves in space plasmas (e.g., Matsumoto and Saito, 1984) and beamplasma interaction (e.g., Umeda, 2010). This brief paper describes instead, initial efforts to develop a fully kinetic HYBem model to expand the usage of the fully kinetic electromagnetic approach to study other terrestrial planets.

This initial brief report on the model is organized as follows. First, basic characteristic of the HYB-em model are described emphasizing its similarities and differences with the HYB-object model. Then two examples are presented in which the properties of the new model are studied: (1) expanding spherical wave generated by a point like source and (2) propagation of a plane wave in a non-magnetized plasma. Finally, advantages and limitations of the developed model are discussed.

\section{Description of the HYB-em model}

HYB-em is from the technical point of view a modified version of the previous self-consistent HYB models which give global plasma and magnetic field values around an object in a plasma flow. The flowing plasma can either be solar wind plasma (Mercury, Venus, the Moon, Mars, asteroids) or magnetospheric plasma (Titan in Saturn's magnetic field).

The HYB platform can be used to study both high densityhigh magnetic field plasma environments, such as Mercury, and low density-low magnetic field plasma environments, such as Mars and Venus. All these three terrestrial planets are scientific objectives for the HYB models. Two examples of the solution based on the previous hybrid HYB models are shown in Fig. 1: Mercury and Mars. The HYB model is described in detail elsewhere (e.g. see Kallio and Janhunen, 2003, for details of the HYB-Mercury model and Kallio et al., 2010, for the HYB-Mars model). In this paper we only summarize the basic properties of the HYB-em model and how it differs from the HYB-object model.

\subsection{The HYB-em model}

The HYB-em model is a Particle-in-Cell (PIC) simulation, similarly as the other HYB models.

In a PIC model particles are modeled as a finite volume clouds where as the electric and magnetic fields are saved and propagated on cells in a grid. Particle clouds are accumulated into the cells and, consequently, every grid cell contains the charge density and the bulk velocity of all particle species in the simulation. In the HYB-em model analyzed in this paper, particles (ions and electrons) are modelled as cube shape "clouds" with homogenous charge density and the same size as the grid cell of volume $\Delta V$. A macroparticle gives a contribution to all cells that it overlaps. In the volume-weighting scheme the charge assignment is performed as follows: if $\Delta V_{\mathrm{p}}^{j}\left(\mathbf{r}_{k}\right)$ is the volume of a particle $j$ of a type $p$ of a weight $w_{\mathrm{p}}^{j}$ which overlaps a grid cell at the point $\mathbf{r}=\mathbf{r}_{k}$ of a volume 


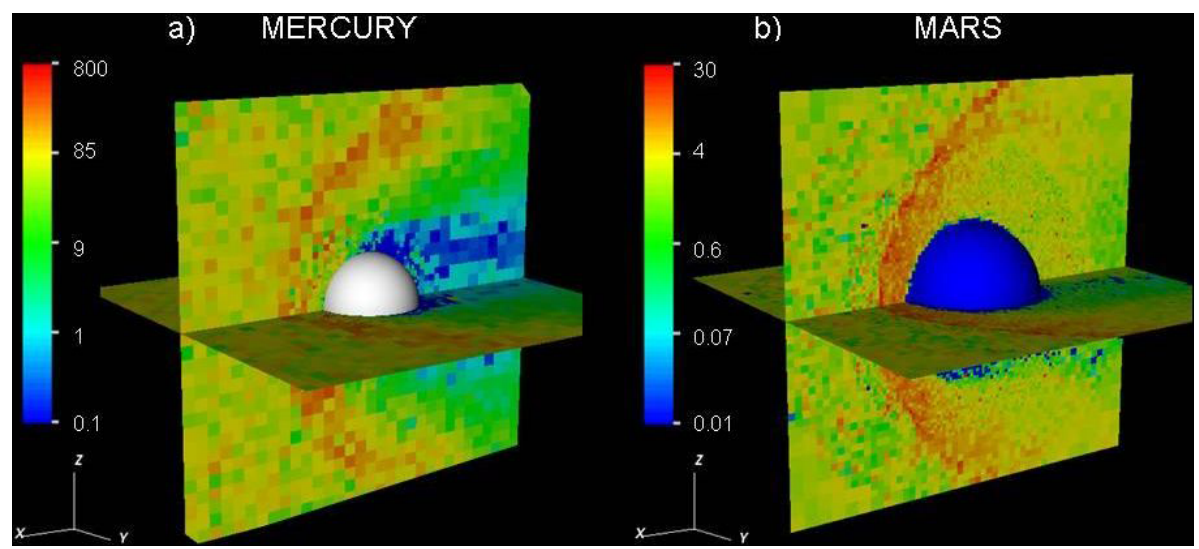

Fig. 1. Planetary plasma environments in the HYB model: (a) Mercury based on the HYB-Mercury model and (b) Mars based on the HYB-Mars model. The colors in the $y=0$ and $z=0$ planes gives the density of the solar wind protons in $\mathrm{cm}^{-3}$. The radius of Mercury in panel (a) is $2440 \mathrm{~km}$ and Mars in (b) is $3393 \mathrm{~km}$. In the figure the solar wind is flowing from left to right and it forms a bow shock around the planets. These plasma parameters from HYB models can automatically be used as input parameters in the HYB-em model.

$\Delta V$, then the grid cell contains

$\Delta w_{\mathrm{p}}^{j}\left(\mathbf{r}_{k}\right)=w_{\mathrm{p}}^{j} \Delta V_{\mathrm{p}}^{j}\left(\mathbf{r}_{k}\right) / \Delta V$

particles due to the macroparticle $j$. The particle density, $n_{\mathrm{p}}$, and the particle bulk velocity, $\mathbf{U}_{\mathrm{p}}$, are determined in a grid cell by the equations

$$
\begin{aligned}
n_{\mathrm{p}}\left(\mathbf{r}_{k}\right) & =\frac{\sum_{j} \Delta w_{\mathrm{p}}^{j}\left(\mathbf{r}_{k}\right)}{\Delta V} \\
\mathbf{U}_{\mathrm{p}}\left(\mathbf{r}_{k}\right) & =\frac{\sum_{j} \Delta w_{\mathrm{p}}^{j}\left(\mathbf{r}_{k}\right) \mathbf{v}_{\mathrm{p}}^{j}}{\sum_{j} \Delta w_{\mathrm{p}}^{j}\left(\mathbf{r}_{k}\right)} .
\end{aligned}
$$

Here the sum $j$ is over all particles, that overlap the grid cell under consideration and that are of type $p$, where the type stands either for an electron or a species of ion. $\Delta V$ is the volume of the grid cell and $\mathbf{v}_{\mathrm{p}}^{j}$ is the velocity of the macroparticle $j$ of type $p$.

The magnetic field, $\boldsymbol{B}$, is propagated in HYB-em and the other HYB models using Faraday's law and the electric field $E$ :

$\partial \boldsymbol{B} / \partial t=-\nabla \times \boldsymbol{E}$

The propagation of $\boldsymbol{B}$ is performed in all HYB models by using a staggered Yee type grid, where the magnetic field is saved on the cell faces. This method keeps the divergence of the magnetic field at zero when the initial magnetic field was divergence free, that is,

$$
\nabla \cdot \boldsymbol{B}=0
$$

is fulfilled automatically in all HYB models.

In the HYB-em model the electric field is a dynamic variable in a similar way as the magnetic field. The E-field is an independent variable and its value is saved on the cell edges. The E- and B-fields are thus organized in a Yee type grid. The E-field is propagated using Ampère-Maxwell's law which contains the displacement current term, $\mu_{o} \varepsilon_{o} \partial \boldsymbol{E} / \partial t$ :

$\partial \boldsymbol{E} / \partial t=\nabla \times \boldsymbol{B} / \varepsilon_{o} \mu_{o}-\mathbf{j} / \varepsilon_{o}$

Here $\boldsymbol{j}, \varepsilon_{o}$ and $\mu_{o}$ are the electric current, the electric permittivity and the magnetic permeability. The electric current in Eq. (6) is derived from macroscopic plasma parameters:

$\mathbf{j}=\sum_{i}\left(q_{i} n_{i} \mathbf{U}_{i}\right)-e n_{e} \mathbf{U}_{e}$

where $\mathbf{U}_{e}, n_{e}$ and $e$ are the bulk velocity of the electrons, the density of the electrons and the elementary charge $\left(1.6 \times 10^{-19} \mathrm{C}\right)$. In Eq. (7) the sum is over all ion species $i$ which particle density, bulk velocity and electric charge is $n_{i}, \mathbf{U}_{i}$ and $q_{i}$, respectively. As already noted, in the HYB-em model both ions and electrons are modeled as particle clouds. The densities and bulk velocities needed to derive the electric current in Eq. (7) is obtained by accumulating particle clouds to the grid cells according to Eqs. (2) and (3). Note that Eq. (6) is similar to Eq. (4) with an exception of $\mathbf{j} / \mu_{o}$ term. For example, in vacuum, $\mathrm{j}$ is zero and the relation between $\boldsymbol{E}$ and $\boldsymbol{B}$ in Eq. (6) is similar to the relation between $\boldsymbol{B}$ and $\boldsymbol{E}$ in Eq. (4).

In the HYB-em model the propagation of $\boldsymbol{B}$ and $\boldsymbol{E}$ in Eqs. (4) and (6) is performed by a contour path (CP) method (see e.g., Jurgens et al., 1992). The method by which Maxwell's four equations are solved is a Finite-Difference Time-Domain (FDTD) (see e.g., Taflove and Brodwin, 1975) method combined with particle propagation.

An important feature in the HYB-em model is that one does not need to solve Maxwell's first equation, Gauss's law

$\nabla \cdot \boldsymbol{E}=\rho / \varepsilon_{o}$ 
where $\rho$ is the total charge density. In the HYB-em model Gauss's law is fulfilled if it is fulfilled initially and if the equation of continuity

$\nabla \cdot \mathbf{j}=-\partial \rho / \partial t$

is satisfied. This conclusion can be obtained by taking divergence from Eq. (6) and by then using the equation of continuity, Eq. (9), which gives

$\partial\left(\nabla \cdot \boldsymbol{E}-\rho / \varepsilon_{\mathbf{o}}\right) / \partial t=0$

Equation (10) shows that Gauss's law is satisfied during the run if it holds initially and if the simulation conserves charge. The accuracy of the method relies therefore on how accurately the charge density and current density obeys Eq. (9). As already mentioned, in this paper we use the volume weighted charge and electric current accumulation scheme as in the previous HYB models. More accurate particle accumulation algorithms exist (see e.g. Villasenor and Buneman, 1992, or Umeda et al., 2003) and they will be tested in the HYB-em in the future.

Finally, the particles in the HYB-em model are accelerated by the Lorentz force:

$$
\begin{aligned}
& m_{i} d \mathbf{v}_{i} / d t=q_{i}\left(\boldsymbol{E}-\mathbf{v}_{i} \times \boldsymbol{B}\right) \\
& m_{e} d \mathbf{v}_{e} / d t=-e\left(\boldsymbol{E}-\mathbf{v}_{e} \times \boldsymbol{B}\right)
\end{aligned}
$$

Here $m_{i}, \boldsymbol{v}_{i}, m_{e}$ and $\boldsymbol{v}_{e}$ are the mass of ion species $i$, the velocity of ion of species $i$, the mass of electron and the velocity of an electron, respectively.

\subsection{The HYB-em model versus a hybrid model}

It is illustrative to look in more detail in how HYB-em differs from the previous HYB models. The magnetic field is firstly the only propagated field variable in the previous HYB models and it is propagated by Faraday's law (Eq. 4) using a CP method in a similar way as in HYB-em.

The electric field is secondly derived in the previous HYB models from the electron fluid momentum equation by adopting a massless electron approximation:

$\boldsymbol{E}=-\mathbf{U}_{e} \times \boldsymbol{B}-\nabla p_{e} /\left(n_{e} e\right)$

Here $p_{e}$ is the electron pressure which is commonly derived by assuming that the electron plasma is isothermal (i.e. $T_{e}=$ const.) or assuming adiabatic electron plasma ( $p_{e} / n_{e}^{\gamma}=$ const. where $\gamma$ is the adiabatic constant). The ion number density, $n_{i}$ and bulk velocity, $\mathbf{U}_{i}$ are derived in a similar way in HYB-em as in the other HYB models from Eqs. (2) and (3). The electron number density, $n_{e}$ and bulk velocity $\mathbf{U}_{e}$ are, however, obtained differently. This is because electrons are modeled as a fluid and not as particles in the earlier HYB models. The electron density is derived in the other HYB models from the ion charge densities by assuming quasi-neutrality

$n_{e}=\sum_{i}\left(q_{i} n_{i}\right) / e$ that is, the electron charge density is assumed to be equal to the total charge density of ions. The electron bulk velocity, $\mathbf{U}_{e}$, is derived from the definition of the electric current (Eq. 7) where $\mathbf{j}$ in turn is derived from the magnetic field by using Ampère-Maxwell's law without the displacement current:

$\mathbf{j}=\nabla \times \boldsymbol{B} / \mu_{o}$

When Eqs. (13) and (14) are combined with Eq. (12) the electric field gets the form

$$
\begin{aligned}
\boldsymbol{E}= & -\sum_{i}\left(q_{i} n_{i} \mathbf{U}_{i}\right) \times \boldsymbol{B} /\left(n_{e} e\right)+(\nabla \times \boldsymbol{B}) \times \boldsymbol{B} /\left(\mu_{o} n_{e} e\right) \\
& -\nabla p_{e} /\left(n_{e} e\right)
\end{aligned}
$$

Equation (15) implies that $\boldsymbol{E}$ can be derived from the macroscopic parameters of the ions and the magnetic field. The electric field is therefore not an independent variable in the HYB-object model. Finally, the ions are propagated using the Lorentz force (Eq. 11a).

It is important to note that HYB-em is a stand-alone model. However, the main advantage of building HYB-em on the same platform as the previous HYB simulations comes from the fact that the initial plasma and field values corresponding to self-consistent stationary or non-stationary solutions from the earlier models are automatically available as initial conditions to HYB-em. The role of the initial conditions is crucial as the above discussions of the validity of Gauss's law shows. The initial conditions must be selfconsistent for the simulation to produce correct results. It is therefore natural to use HYB-em with the earlier HYB models. An obvious way to use HYB-em in conjunction with an earlier HYB model is to choose initial plasma and field values from a stationary or non-stationary solution and to study possible generation of instabilities, associated waves and their propagation in 3-D plasma. Initial parameters, describing both stationary and non-stationary initial conditions can, of course, also be obtained from other models or from observations.

\section{Electromagnetic waves}

In this section we study the applicability of the developed model by analyzing two examples of the propagation of electromagnetic waves. The examples, namely (1) an oscillating 3-D point source and (2) an oscillating 1-D plane source, were chosen because the numerical solutions can be compared with analytical solutions which give at least the first order solution for the analyzed problems.

In all presented runs the time step, $d t$, is $1 \times 10^{-5} \mathrm{~s}$ and the frequency of the oscillating source, $f_{\text {source }}$, is $1.5 \mathrm{kHz}$. These parameters imply that the wave length of the em-wave in vacuum, $\lambda_{\text {vacuum }}\left(=c / f_{\text {source, }}\right.$ where $c$ is the speed of the light in a vacuum), is $200 \mathrm{~km}$. In all runs the magnetic field, the electric field and the bulk velocities are initially zero. Moreover, in all presented runs the grid size, $d x$, was $10 \mathrm{~km}$. 


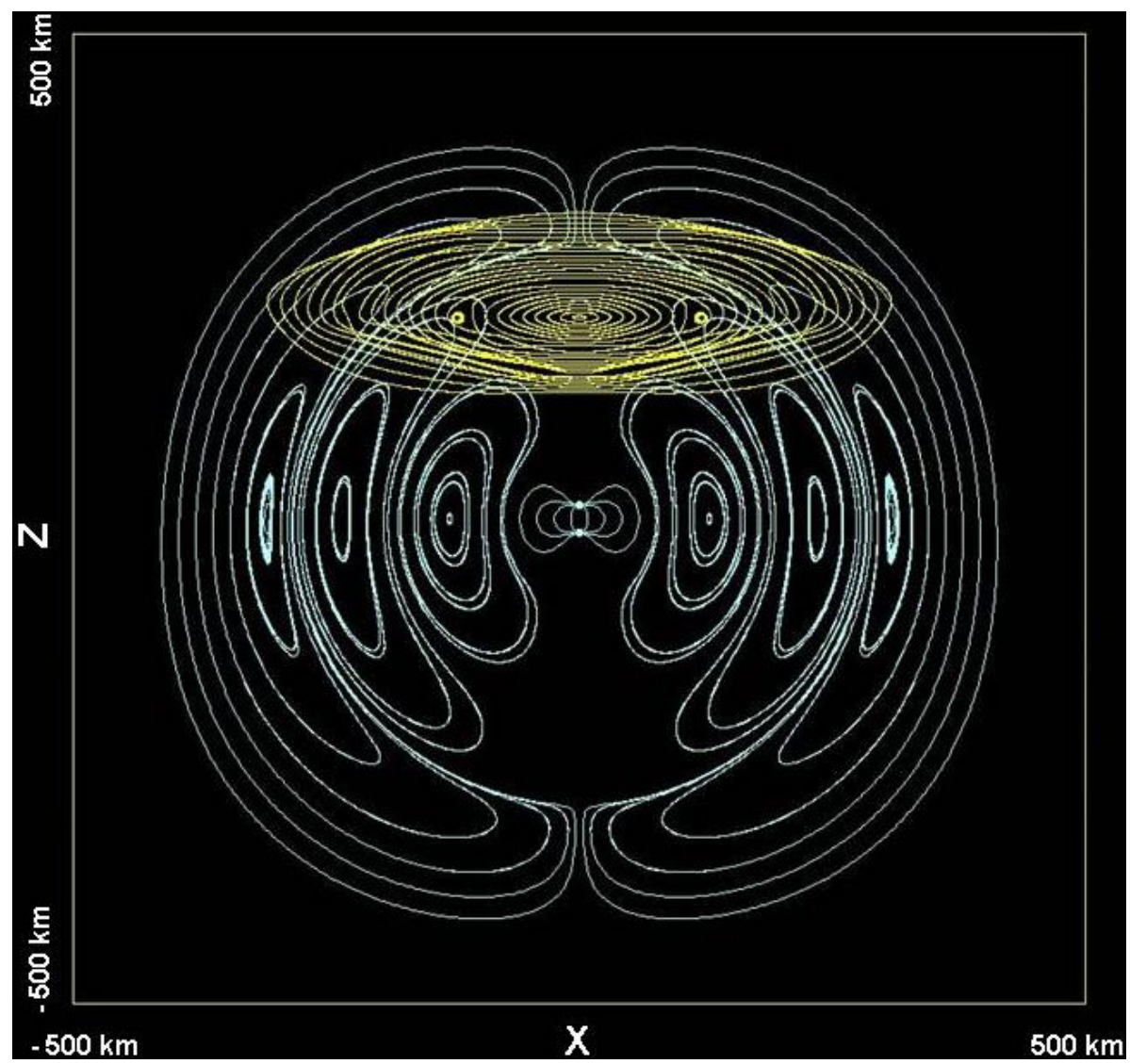

Fig. 2. A 3-D spherical wave in the HYB-em model. The run includes an oscillating point source in a vacuum located at the center of the figure. The source generates a sinusoidal electric field with a frequency of $1.5 \mathrm{kHz}$. The white lines and the yellow lines are the electric and the magnetic field lines, respectively, which are added to illustrate the electromagnetic field in 3-D. The horizontal axis and the vertical axis are the $\mathrm{x}$ - and the $\mathrm{z}$-axis, respectively. The size of the box is $-500 \mathrm{~km}<x, y, z<500 \mathrm{~km}$. Note that the vantage point is slightly (10 deg.) below the $z=0$ plane in order to see the circular B-lines about the $\mathrm{z}$-axis.

\subsection{Oscillating point source}

In the first run an oscillating point like source is placed into the vacuum. The size of the simulation box is $-500 \mathrm{~km}<$ $x, y, z<500 \mathrm{~km}$ and the source was positioned at the center of the box. The electric field at the point source is varied manually as

$\boldsymbol{E}(t)=\left(E_{x}, E_{y}, E_{z}\right)=E \times(0,0, \sin (2 \pi(t / T)))$

from the beginning of the simulation at $t=0 \mathrm{~s}$. Here $E$ is the amplitude of the electric field at the point source which in the test is $3 \times 10^{-4} \mathrm{~V} / \mathrm{m}$. The simulation contains 100 grids cells in the $x^{-}, y_{-}$, and $\mathrm{z}$-directions.

Figure 2 shows a snapshot of the result in 3-D. It gives an example of electric and magnetic field lines in the simulation box at $t=1.12 \mathrm{~ms}$ when the em-wave fills the whole simulation box. As can be seen in Fig. 2, the solution is symmetric about the $\mathrm{z}$-axis including circular magnetic field lines about the $\mathrm{z}$-axis and toroidal-like electric field lines around B-lines, as the analytic solution suggests (see e.g., Alonso and Finn, 1980). The phase speed of the wave is the speed of light, the wavelength is $\lambda_{e m}$ and the ratio of the maximum electric field and the maximum magnetic field, $\max (\boldsymbol{E}) / \max (\boldsymbol{B})$, is the speed of light, implying that the propagating disturbance is an em-wave. The test suggests that the derived HYB-em model is anticipated to be able to capture basic properties of the 3-D em-wave in the HYB model platform.

\subsection{Propagation of a plane wave}

In the second set of runs an oscillating plane source is firstly placed into the vacuum at $x=0$ plane. The size of the simulation box at all three plane wave runs is $-2500 \mathrm{~km}<x<$ $2500 \mathrm{~km}$ and $-50 \mathrm{~km}<y, z<50 \mathrm{~km}$, that is, 500 grid cells in the $\mathrm{x}$-direction and 10 cells in the $\mathrm{y}$ - and $\mathrm{z}$-directions. The size of the simulation box in the $\mathrm{x}$-direction is, therefore, about the same as the $\mathrm{x}$-scale in the other hybrid HYB runs shown in Fig. 1. The source is a plane where the value of the 


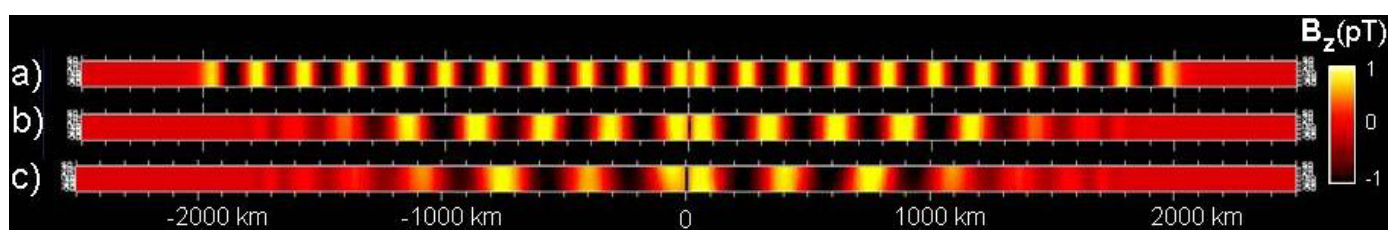

Fig. 3. Plane wave runs in the HYB-em model: The magnetic field. The oscillating plane source is located at $x=0$ and its frequency is $1.5 \mathrm{kHz}$. The colors in panels $(\mathbf{a}-\mathbf{c})$ show the $\mathrm{z}$ component of the magnetic field from $-1 \times 10^{-12} T(=-1 \mathrm{pT})$ to $1 \times 10^{-12} T(=1 \mathrm{pT})$ at $t=6.96 \mathrm{~ms}$, i.e. just before the plane wave reaches the faces of the simulation box at $x= \pm 2500 \mathrm{~km}$. In all panels the horizontal axis is the $\mathrm{x}$-axis from $-2500 \mathrm{~km}$ to $+2500 \mathrm{~km}$, and the vertical axis corresponding to the $\mathrm{z}$-axis from $-50 \mathrm{~km}$ to $+50 \mathrm{~km}$. The magnetic field is derived at the $y=0$ panel. Panel (a) shows the electromagnetic plane wave in a vacuum. In panels (b) and (c) the simulation box was filled initially with the plasma of electron density of $\sim 0.016 \mathrm{~cm}^{-3}$ and $\sim 0.021 \mathrm{~cm}^{-3}$, respectively.

magnetic field was varied manually as

$\boldsymbol{B}(t)=\left(B_{x}, B_{y}, B_{z}\right)=B_{o} \times(0,0, \sin (2 \pi(t / T)))$

The amplitude of the magnetic field, $B_{o}$, is taken to be $0.001 \mathrm{nT}(=1 \mathrm{pT})$. Periodic boundary conditions are implemented both for the plasma and the fields at $z= \pm 50 \mathrm{~km}$ faces. The electromagnetic wave is shown at $t=6.96 \mathrm{~ms}$ when the wave does not fill the whole simulation box and the wave is still approaching the $\pm x$ faces of the simulation box.

The plane wave runs are performed both for a vacuum situation and for a situation where the simulation box contains an electron-proton plasma. Figure 3 a shows the result based on the first plane wave test when the source is in a vacuum. We learn from Fig. 3a, that the HYB-em model produces successfully a plane wave in a vacuum with the correct wavelength, that it keeps the wave amplitude practically unchanged while the wave is propagating along the $\mathrm{x}$-axis, and that the wave propagates away from the source with speed of the light. In this run, the value of $\max (\boldsymbol{E}) / \max (\boldsymbol{B})$ is close the speed of light, providing a further evidence that the propagating disturbance is an electromagnetic wave.

In the second and third plane wave runs the simulation box is filled with plasma. The plasma contains an equal amount of $\mathrm{H}^{+}$ions and electrons. The ions and electrons are initially at rest, that is, their bulk velocity and temperature are zero. The initial positions are chosen randomly in the simulation box. There are on average 30 macro particles per grid cell. In the second plane wave run the electron density is $15505 \mathrm{~m}^{-3}$, i.e. $\sim 0.016 \mathrm{~cm}^{-3}$. This corresponds to the electron plasma frequency, $f_{p e}=\sqrt{e^{2} n_{e} /\left(m_{e} \varepsilon_{o}\right)} /(2 \pi)$ $\left(\sim 8.98 \mathrm{kHz} \sqrt{n_{e}}\right.$ where $n_{e}$ is unit of $\left.\mathrm{cm}^{-3}\right)$, of $1.118 \mathrm{kHz}$. In the third plane wave run the electron density is increased to $20932 \mathrm{~m}^{-3}\left(\sim 0.021 \mathrm{~cm}^{-3}\right)$ which corresponds to a $f_{p e}$ of $1.299 \mathrm{kHz}$.

Figure $3 \mathrm{~b}$ shows that also in this run the wave is propagating into the plasma with approximately constant wavelength and amplitude. However, we see that the wavelength is now longer than in the vacuum case (cf. Fig. 3a). Moreover, when the electron density is increased even more in the third run (Fig. 3c) the wavelength is increased once again.
In Fig. 4 the variations of the plasma parameters are shown. We learn the following from Fig. 4a. The electron density and the proton density are firstly quite similar in the whole simulation box, that is, both near the source where the wave is fully developed and near the $\pm x$ faces where the plasma is still practically undisturbed. Secondly, the velocity of electrons are much higher than the velocity of ions the maximum velocity ratio $\max \left|U_{y}\left(e^{-}\right)\right| / \max \left|U_{y}\left(\mathrm{H}^{+}\right)\right|$ being about the same as their mass ratio $m_{p} / m_{e}(\sim 1863)$. Thirdly, the solution seen in Fig. $4 \mathrm{~b}$ resembles qualitatively the solution of the second plane wave run (Fig. 4a) but a similar kind of increase in the wave length of the disturbances in the plasma parameters can be identified, as in the magnetic field.

The wave length continues increasing when the electron density is increased even more(figures not shown). But when the electron density was increased to $27901 \mathrm{~m}^{-3}$ $\left(f_{p e}=1.5 \mathrm{kHz}\right)$ and in the runs when the density is even higher, the solution started to change dramatically: the wave does not any more propagate properly away from the plane source, but it looks like part of the wave is reflected back from the plasma toward the plane source finally destroying the run (figures not shown).

One may try to understand the results of the plane wave runs in Figs. 3 and 4 by comparing them to results given by the linear theory of electromagnetic waves in nonmagnetized plasma. One may, indeed, expect this theory to give a first order approximation to the results in the runs because the theory is based on the following assumptions which are good approximations also in the analyzed runs (cf. Fig. 4): (1) the density of plasma is near its initial value, (2) the velocity of electrons are much higher than the velocity of ions. In this kind of situation the electrons carry the electric current, that is, $\mathbf{j} \sim e n_{o}\left(e^{-}\right) \mathbf{U}\left(\mathrm{e}^{-}\right)$, where $n_{o}\left(e^{-}\right)$is the initial electron density. In other words, the wave frequency is so high that the plasma fluctuations are mainly associated with electron velocity fluctuations. 


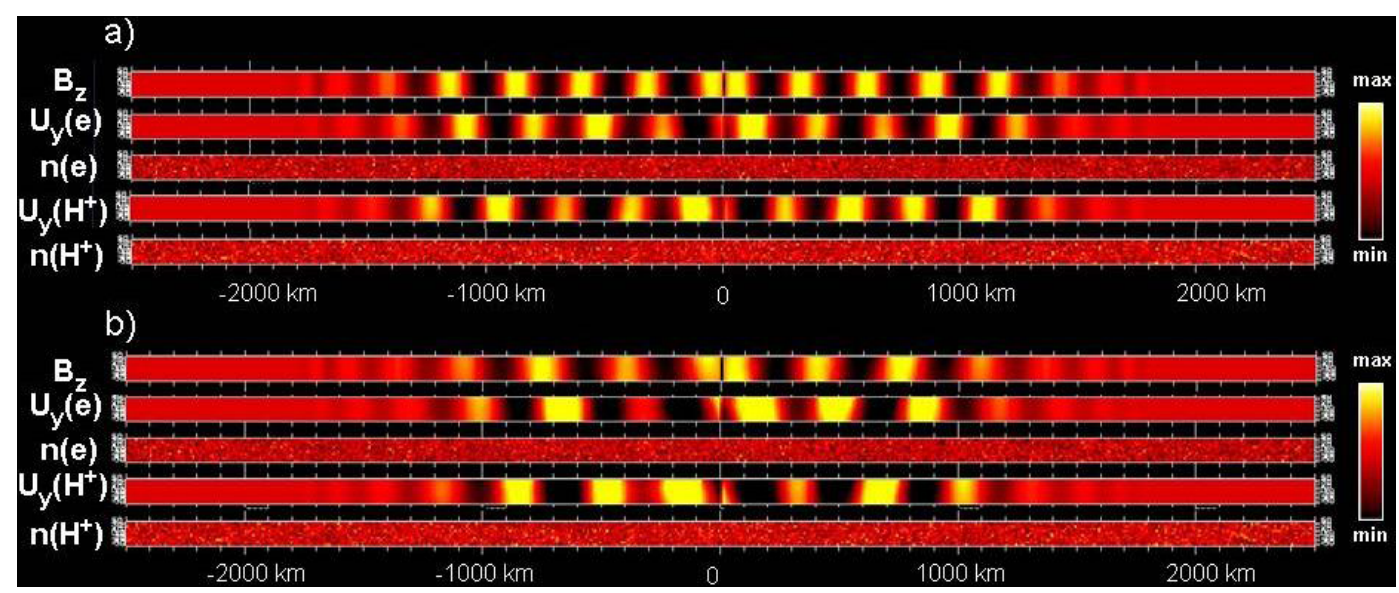

Fig. 4. Plane wave runs in the HYB-em model: Plasma parameters. In panel (a) the initial electron density in the simulation box was $\sim 0.016 \mathrm{~cm}^{-3}$ and in panel (b) it was $\sim 0.021 \mathrm{~cm}^{-3}$. The axes are as in Fig. 3: The horizontal axis is the $\mathrm{x}$-axis from $-2500 \mathrm{~km}$ to $+2500 \mathrm{~km}$, and the vertical axis from $-50 \mathrm{~km}$ to $+50 \mathrm{~km}$. The values are created at the $y=0$ plane. The oscillating plane source is positioned at $x=0$ and its frequency is $1.5 \mathrm{kHz}$. The values correspond to the situation at $t=6.64 \mathrm{~ms}$. In panel (a) the tables from top to bottom are: $B_{z}$ from $-1 \times 10^{-12} \mathrm{~T}$ to $1 \times 10^{-12} \mathrm{~T}$, the velocity of electrons in the y-direction $\left(U_{y}(e)\right)$ from $-10 \mathrm{~km} / \mathrm{s}$ to $10 \mathrm{~km} / \mathrm{s}$, the electron density $(n(e))$ from 0 to $0.03 \mathrm{~cm}^{-3}$, the velocity of protons in the y-direction $\left(U_{y}\left(\mathrm{H}^{+}\right)\right)$from $-5 \mathrm{~m} / \mathrm{s}$ to $5 \mathrm{~m} / \mathrm{s}$ and the proton density $\left(n\left(\mathrm{H}^{+}\right)\right)$from 0 to $0.03 \mathrm{~cm}{ }^{-3}$. In panel (c) the initial electron density was increased to $0.021 \mathrm{~cm}^{-3}$. Tables from top to bottom are: $B_{z}$ from $-1 \times 10^{-12} \mathrm{~T}$ to $1 \times 10^{-12} \mathrm{~T}$, $U_{y}(e)$ from $-10 \mathrm{~km} / \mathrm{s}$ to $10 \mathrm{~km} / \mathrm{s}, n(e)$ from 0 to $0.04 \mathrm{~cm}^{-3}, U_{y}\left(\mathrm{H}^{+}\right)$from $-5 \mathrm{~m} / \mathrm{s}$ to $5 \mathrm{~m} / \mathrm{s}$ and $n\left(\mathrm{H}^{+}\right)$from 0 to $0.04 \mathrm{~cm}^{-3}$. In both panels the color map on the right hand side is a linear color map from the minimum value (min) of the variable to the maximum value (max) of the variable.

The linear theory shows that the dispersion relation of high frequency em-waves is (see e.g., Schunk and Nagy, 2004)

$\omega^{2}=\omega_{p e}^{2}+c^{2} k^{2}$

where $\omega=2 \pi / T$ is the angular frequency of the wave, $\omega_{p e}$ is the electron plasma frequency, $c$ is the speed of light and $k=2 \pi / \lambda$ is the wave vector, where $T$ and $\lambda$ are the wave period and wave length, respectively. The wave length of a propagating wave is therefore

$\lambda=2 \pi c / \sqrt{\omega^{2}-\omega_{p e}^{2}}=c / \sqrt{f^{2}-f_{p e}^{2}}$.

According to Eq. (19) the wavelengths in the three cases shown in Fig. 3a (vacuum), 3b $\left(f_{p e}=1.118 \mathrm{kHz}\right)$ and $3 \mathrm{c}$ $\left(f_{p e}=1.299 \mathrm{kHz}\right)$ are $200 \mathrm{~km}, 300 \mathrm{~km}$ and $400 \mathrm{~km}$, respectively, which are relatively good approximations for the simulated wave lengths. The dramatic change of the characteristics of the em-wave in the runs where the electron density exceeds $27901 \mathrm{~m}^{-3}$ can also be understood on the basis of Eq. (18). In these densities the electron plasma frequency is higher than the frequency of the plane source resulting in the damping of the wave and reflection of the wave energy back against the plane wave source.

\section{Discussion}

This paper describes a fully kinetic electromagnetic model developed for planetary space plasma physical research, aimed especially to study instabilities and electromagnetic waves. Space plasma environments which are natural targets for the forthcoming work based on HYB-em are the plasma environments of Mercury, Venus and Mars. The goal of the work is to develop a fast, robust, well tested numerical 3-D model for both basic plasma physical research and, especially, to (1) interpret the ongoing plasma and field observations at Mars and Venus by the Mars Express and the Venus Express missions and to (2) analyze physical processes which will be measured at Mercury by BepiColombo mission.

The newly developed HYB-em model is based on the preexisting HYB models. HYB-em is consequently built on a platform which is robust, computationally effective and well tested due to a ten year long development and testing period of the HYB platform. Another important advantage of this close relation to the earlier HYB models is that selfconsistent initial 3-D plasma and field values obtained from the other global HYB models can automatically be adopted as inputs to HYB-em. In addition, all physical processes in a multi ion species plasma that are currently implemented on the HYB platform, such as ion-neutral collisions, electron impact ionization, charge exchange processes and photoionization are automatically available also in HYB-em.

Electromagnetic waves provide one natural class of physical phenomena that can be studied with HYB-em. Wave phenomena in space plasmas contain an extremely rich set of physical processes. This is partly due to the fact that the 
interaction between plasmas and em-waves is a complex matter and partly due to that the properties of the plasma environments vary substantially in the Solar System in terms of the plasma density, strength of the magnetic field and by the density of neutral particles. Waves also carry electromagnetic energy which can be transferred onto the particles and vice versa. Wave phenomena is a subject of continuous interests in space plasma physics and the Solar System objects are under the influence of the supersonic and super-alfvenic flow of the solar wind.

Modeling of em-waves in planetary plasma environments has a long history but it is still a challenging task because properties of the waves depend on the properties of the plasma (density, velocity and temperature), the magnetic field (its strength and the direction of the wave with respect to the direction of the magnetic field), possible collisions of charged particles (with charged particles or neutrals), how the wavelength is related to the characteristic length scales in the plasma (especially, to the Debye length), how the frequency of the wave is related to the characteristic time scales in the plasma (e.g. plasma frequencies, collision frequencies and gyro frequencies) as well as the electric properties of the solid objects in the plasma (Mercury, asteroids etc.). The developed HYB-em model has a potential to study all these complicated plasma physical processes.

\section{Summary}

A fully kinetic electromagnetic particle model has been developed to study planetary plasma environments. The threedimensional (3-D) model, HYB-em, can be used in conjunction with a 3-D hybrid model which is developed to study how the solar wind interacts with various Solar System objects. The preliminary studies made by the HYB-em model suggest that the concept is mature in the HYB model platform and that it can be used to interpret ongoing plasma and field measurement at Mars and Venus and the forthcoming measurements at Mercury.

Acknowledgements. The authors are indebt to Riku Jarvinen and Pekka Janhunen at the Finnish Meteorological Institute for their crucial role in the development of the HYB modeling platform on which the main functionalities of the HYB-em model are based. The authors also thank Riku Jarvinen and Kaijun Liu for valuable comments concerning the HYB-em model and the manuscript.

Topical Editor R. Nakamura thanks one anonymous referee for her/his help in evaluating this paper.

\section{References}

Alonso, M. and Finn, E. J.: Fundamental University Physics II: Fluids and Waves, Addison-Wesley publishing company, 1980.

Blomberg, L. G., Matsumoto, H., Bougeret, J.-L., Kojima, H., Yagitani, S., Cumnock, J. A., Eriksson, A. I., Marklund, G. T., Wahlund, J.-E., Bylander, L., Åhlén, L., Holtet, J. A., Ishisaka,
K., Kallio, E., Kasabai, Y., Matsuoka, A., Moncuquet, M., Mursula, K., Omura, Y., and Trotignon, J. G.: MEFISTO - An electric field instrument for BepiColombo/MMO, Adv. Space Res., 38(4), 672-679, 2006.

Brain, D., Barabash, S., Boesswetter, A., Bougher, S., Brecht, S., Chanteur, G., Crider, D., Dubinin, E., Fang, X., Fraenz, M., Halekas, J., Harnett, E., Holmstrom, M., Kallio, E., Lammer, H., Ledvina, S., Liemohn, M., Liu, K., Luhmann, J., Ma, Y., Modolo, R., Motschmann, U., Nilsson, H., Shinagawa, H., and Terada, N.: A Comparison of Global Models for the Solar Wind Interaction with Mars, Icarus, 206(1), doi:10.1016/j.icarus.2009.06.030, 139-151, 2010.

Buneman, O., Barnes, C. W., Green, J. C., and Nielsen, D. E.: Principles and capabilities of 3-d, E-M particle simulations, J. Comput. Phys., 38(1), 1-44, 1980.

Duru, F., Gurnett, D. A., Morgan, D. D., Modolo, R., Nagy, A. F., and Najib, D.: Electron densities in the upper ionosphere of Mars from the excitation of electron plasma oscillations, J. Geophys. Res., 113, A07302, doi:10.1029/2008JA013073, 2008.

Ergun, R. E., Andersson, L., Peterson, W. K., Brain, D., Delory, G. T., Mitchell, D. L., Lin, R. P., and Yau, A. W.: Role of plasma waves in Mars' atmospheric loss, Geophys. Res. Lett., 33, L14103, doi:10.1029/2006GL025785, 2006.

Espley, J. R., Cloutier, P. A., Brain, D. A., Crider, D. H., and Acuña, M. H.: Observations of low frequency magnetic oscillations in the Martian magnetosheath, magnetic pileup region, and tail, J. Geophys. Res., 109, 7213, doi:10.1029/2003JA010193, 2004.

Grigoriev, A., Futaana, Y., Barabash, S., and Fedorov, A.: Observations of the Martian subsolar ENA jet oscillations, Space Sci. Rev., 126, 299-313, doi:10.1007/s11214-006-9121-y, 2007.

Jurgens, T. G., Umashankar, K., and Moore, T. G.: FiniteDifference Time-Domain modelling of curved surfaces, IEEE Transactions of antennas and propagation, 40(4), 357-366, 1992.

Kallio, E., Koskinen, H., Barabash, S., Nairn, C. M. C., and Schwingenschuh, K.: Oxygen outflow in the Martian magnetotail, Geophys. Res. Lett., 22(18), 2449-2452, 1995.

Kallio, E. and Janhunen, P.: Modelling the solar wind interaction with Mercury by a quasi-neutral hybrid model, Ann. Geophys., 21, 2133-2145, 2003, http://www.ann-geophys.net/21/2133/2003/.

Kallio, E., Liu, K., Jarvinen, R., Pohjola, V., and Janhunen, P.: Ion escape at Mars in a hybrid model: high energy and low energy ions, Icarus, 206(1), 152-163, doi:10.1016/j.icarus.2009.05.015, 2010.

Matsumoto, H. and Saito, T.: Particle simulation of electromagnetic waves and its application to space plasmas, in Computer simulation of space plasmas, Terra Scientific Publishing Company, 43-102, 1984.

Nielsen, E., Wang, X.-D., Gurnett, D. A., Kirchner, D. L., Huff, R., Orosei, R., Safaeinili, A., Plaut, J. J., and Picardi, G.: Vertical sheets of dense plasma in the topside Martian ionosphere, J. Geophys. Res., 112, E02003, doi:10.1029/2006JE002723, 2007.

Nishikawa, K.-I.: Tristan code and its applications, Proceedings of ISSS-6, 1-4, Copernicus Gesellschaft, 2001.

Pernice, W. H. P., Payne, F. P., and Gallagher, D. F. G.: Finitedifference time-domain simulation of dispersive features smaller than the grid spacing, Int. J. Numer. Model., 20, 311-326, doi:10.1002/jnm.648, 2007.

Russell, C. T., Zhang, T. L., Delva, M., Magnes, W., Strange- 
way, R. J., and Wei, H. Y.: Lightning on Venus inferred from whistler-mode waves in the ionosphere, Nature, 450, 661-662, doi:10.1038/nature05930, 2007.

Schunk, R. W. and Nagy, A. F.: Ionospheres: physics, plasma physics and chemistry, Cambridge University Press, The Pitt Building, Trumpington Street, Cambridge, UK, ISBN 0-52160770-1, 2004.

Sakai, J.-I., Eda, M., Miyazaki, H., and Neubert, T.: Electromagnetic Wave Emission from Kelvin-Helmholtz Instability Region in Pair Plasmas, J. Phys. Soc. Japan, 67(7), 2170-2173, 1998.

Spitkovsky, A.: Particle Acceleration in Relativistic Collisionless Shocks: Fermi Process at Last?, The Astrophys. J., 682(1), L5L8, doi:10.1086/590248, 2008.

Taflove, A. and Brodwin, M. E.: Numerical solution of steady-state electromagnetic scattering problems using the time-dependent Maxwell's equations, IEEE Transactions on Microwave Theory and Techniques, 23, 623-630, doi:10.1109/TMTT.1975.1128640, 1975.

Trotignon, J. G., Dubinin, E., Grard, R., Barabash, S., and Lundin, R.: Martian planetopause as seen by the plasma wave system onboard Phobos 2, J. Geophys. Res., 101(A11), 24965-24977, 1996.

Trotignon, J. G., Grard, R., and Savin, S.: Plasma Wave System Measurementos of the Martian Bow Shock From the Phobos 2 Spacecraft, J. Geophys. Res., 96(A7), 11253-11264, 1991.
Umeda, T., Omura, Y., Tominaga, T., and Matsumoto, H.: A new charge conservation method in electromagnetic particle-in-cell simulations, Computer Physics Communications, 156(1), 73-85, doi:10.1016/S0010-4655(03)00437-5, 2003.

Umeda, T.: Electromagnetic plasma emission during beam-plasma interaction: Parametric decay versus induced scattering, J. Geophys. Res., 115, A01204, doi:10.1029/2009JA014643, 2010.

Villasenor, J. and Buneman, O.: Rigorous charge conservation for local electromagnetic field solvers, Computer Physics Communications, p. 306, 1992.

Winningham, D., Frahm, R. A., Sharber, J. R., Coates, A. J., Linder, D. R., Soobiah, Y., Kallio, E., Espley, J. R., Lundin, R., Barabash, S., Holmström, M., Andersson, H., Yamauchi, M., Grigoriev, A., Scherrer, J. R., Jeffers, S. J., Kataria, D. O., Kozyra, J. U., Luhmann, J. G., Roelof, E. C., Williams, D. J., Livi, S., Curtis, C. C., Hsieh, K. C., Sandel, B. R., Koskinen, H., Säles, T., Riihelä, P., Schmidt, W., Grande, M., Carter, M., Sauvaud, J.-A., Fedorov, A., Thocaven, J.-J., McKenna-Lawler, S., Orsini, S., Cerulli-Irelli, R., Maggi, M., Wurz, P., Bochsler, P., Krupp, N., Woch, J., Fränz, M., Asamura, K., and Dierker, C.: oscillations in the induced martian magnetosphere, Icarus, 182(2), 360-370, doi:10.1016/j.icarus.2005.10.033, 2006. 